commenced on hydroxychloroquine and requires 3-4LO2 during day and Opti flow $6 \mathrm{~L} / \mathrm{min}$ at night. She gets blue with slightest agitation, precipitous desaturations and accompanying hypoxic attacks. She has marked finger clubbing and tachypnoea but no obvious increased work of breathing/chest deformity.

Of note her fist cousin (maternal side) has suspected interstitial lung disease along with Bardet-Biedl, she has CT-changes but no surfactant gene abnormalities. Her maternal grandfather died at 45 years with pulmonary fibrosis, also her maternal great grandmother died young with pulmonary fibrosis. Her two half siblings are healthy.

Conclusion We report a child with SFTPC mutation with interstitial lung disease.

\section{ASSOCIATION BETWEEN THROMBOCYTOSIS AND DISEASE SEVERITY IN CHILDREN WITH BRONCHIOLITIS}

Vanda Pavić*, Helena Tesari Crnković, Andrea Šimić Klarić. Pediatric Clinic, Clinical Hospital Centre, Osijek

\subsection{6/archdischild-2021-europaediatrics.421}

The objective of this study was to investigate the frequency of secondary thrombocytosis in children during RSV (respiratory syncytial virus) bronchiolitis and bronchiolitis caused by other cause. Predictive value of secondary thrombocytosis in relation with severity of clinical features and the outcome of the treatment in bronchiolitis affected children has also been studied.

In this retrospective study, medical records of 136 infants treated at Pediatrics department of County General Hospital Požega were used.

Demographic and other patients' features with bronchiolitis were collected from their medical records. The data was statistically examined.

The mean age of participants was 117,5 day. There were $72(52,9 \%)$ boys and $64(47,1 \%)$ girls. $94(64,1 \%)$ of children had positive rapid antigen testing (immunoassay) for RSV from nasopharyngeal aspirate. Statistically significant association between duration of hospitalization and oxygen application in therapy, with thrombocytosis, was determined with logistic regression. The risk for thrombocytosis was increased with every day of hospitalization by $18,5 \%$, and with the oxygen application in therapy by $33,1 \%$. Need for hospitalization and development of pneumonia statistically do not have an influence on the development of thrombocytosis. There wasn't statistically significant difference in trombocytosis according to RSV infection.

Children with bronchiolitis and thrombocytosis had a more severe course of the disease, which was presented with longer duration of hospitalization and the need for oxygen application in therapy.

\section{MODERN VIEW OF A PATIENT WITH BRONCHOPULMONARY DYSPLASIA}

VA Bondar*, IV Davydova, MA Basargina. National Medical Research Center for Children's Health Federal state autonomous institution of the Russian Federation Ministry of Health

10.1136/archdischild-2021-europaediatrics.422

To analyze the new model of a patient with bronchopulmonary dysplasia (BPD) based on the analysis of 70 case histories of infants hospitalized at the National Medical Research Center for Children's Health Federal state autonomous institution of the Russian Federation Ministry of Health from 2020 to 2021.

Collection and analysis of anamnestic data, as well as clinical and laboratory examination of 70 infants with BPD. The analysis of the results was carried out using STATISTICA 10.0 program.

In total, all studied infants $(\mathrm{n}=70)$ were diagnosed with a new form of bronchopulmonary dysplasia. Among them there were 35 boys $(50 \%)$ and 35 girls (50\%). The mean gestational age was 26,4 weeks [SD, 1.9 weeks]. All premature infants were born with extremely low and very low birth weight $(85,7 \%$ and $14,3 \%$, respectively). The median birth weight was $745 \mathrm{~g}$ (Interquartile range (IQR): 650 - 920). The median Apgar score at the 1st minute was 5 (IQR: $4-5$ ), at the 5 th minute -6 (IQR: 5 - 6). 67 infants were intubated after birth, the median of duration of mechanical ventilation was 21 days (IQR: 6 - 36); CPAP treatment was initiated in 33 children, the median was 8 days (IQR: 5 - 13), BIPAP 39 patients, nasal cannulas - 47 infants, nasal mask - 14, incubator - 19. Median duration of oxygen dependence was 54 days (IQR: 45 - 70]. The period of oxygen dependence had an inverse relationship with anthropometric data and gestational age. All patients received surfactant therapy, postnatal corticosteroids - 32 infants (46\%). In addition, pneumonia was observed in 35 children (50\%), intraventricular hemorrhage in 60 patients (86\%), necrotizing enterocolitis in 34 children (49\%). Pulmonary hypertension, as one of the complications of BPD, occurred only in 7 patients (10\%).

The current model of a patient with a new form of BPD is a premature infant with extremely low or very low body weight, born at a gestational age of no more than 28 weeks. The morphological and functional immaturity of these children, combined with the impact of new resuscitation technologies on their respiratory system, led to the pathomorphosis of BPD and the predominance of a new form in the population. Actually the study of the clinical features and long-term outcomes of BPD is an urgent problem of pediatric pulmonology and requires close attention in the future.

\section{GENETICPREDICTORS OF A NEW FORM OF BRONCHOPULMONARY DYSPLASIA}

VA Bondar*, IV Davydova, MA Basargina, KV Savostyanov, AA Pushkov, IS Zhanin, AG Nikitin. National Medical Research Center for Children's Health Federal state autonomous institution of the Russian Federation Ministry of Health

\subsection{6/archdischild-2021-europaediatrics.423}

Bronchopulmonary dysplasia (BPD) is a multifactorial disease with a significant genetic component. Novel genes and associated pathways may play an important role in susceptibility for the development of bronchopulmonary dysplasia in preterm infants. Our aim was to identify rare genetic variants contributing to the new form of BPD phenotype by full exome sequencing.

Full exome sequencing was performed on 39 DNA samples from patients with moderate and severe new BPD and 30 DNA samples from control group without clinical signs of BPD. After mapping and annotation, each sample showed an average of 40,000 genetic variants with a reading depth of at least $70 \mathrm{x}$. 
All autosomal variants were filtered for allelic frequency $<1 \%$ according to the gnomAD database (version 2.1). Among them, 821 variants were found the most common $(\geq 10 \%)$ in both the control and experimental group. Wherein 280 variants were presented with an alternative allele frequency of more than $10 \%$ in the experimental group, but were found with a lower frequency in the control. Interestingly, 10 of these 280 variants were located in the ZNF717 gene. This gene encodes a Kruppel-associated box (KRAB) zinc-finger protein, which belongs to a large group of transcriptional regulators in mammals and play important roles in various cellular functions, including cell proliferation, differentiation and apoptosis. Extended bioinformatics analysis showed that 34 unique variants were found in the experimental group and were absent in the control group, which may indicate both the characteristics of Russian children with BPD and the insufficient representativeness of the control group and, in turn, requires a more in-depth analysis.

For the first time in Russia, large-scale studies have been carried out to identify the molecular genetic characteristics of Russian children with BPD using full exome sequencing. Our study indicates ZNF717 gene may be relevant in BPD pathogenesis, but further research is required. These preliminary results may contribute to improving knowledge of the pathogenesis of bronchopulmonary dysplasia and targeting therapeutic interventions.

\section{WHAT IS BEHIND A SPONTANEOUS PNEUMOTHORAX?}

Joana Filipe Ribeiro*, Iris Santos Silva, João Virtuoso, Pedro Fernandes, Glória Silva, Rita S Oliveira, Pedro Carvalho. Department of Pediatrics, Hospital Sousa Martins, ULS Guarda

10.1136/archdischild-2021-europaediatrics.424

Introduction/Description Secondary Spontaneous Pneumothorax has its peak incidence in adulthood, occurring as a complication of an underlying lung disease, with variable symptoms at presentation.

A 15-year-old female previously healthy was admitted to the Emergency Department complaining of chest pain on left hemithorax and dyspnoea lasting for 24 hours, associated with a non-productive cough for 1 week. She denied fever, rhinorrhea, trauma, foreign body aspiration, inhalation of drugs, consumption of tobacco or any medication. She had no relevant family history. On admission, she was eupneic, SpO2 $99 \%(\mathrm{FiO} 2$ 21\%) and an absence of vesicular murmur was noted on the left hemithorax. Chest radiography revealed a left side pneumothorax with no mediastinal deviation.

Supplemental oxygen was initiated by a nonrebreathing facemask and the patient was advice to rest. The lack of improvement motivated a pneumothorax drainage by needle aspiration with an initial favourable response. However, 24 hours later a recurrence of the pneumothorax was noted in the control radiography. Chest-CT confirmed this finding and depicted an atelectasis of the left lower lobe, with a slight right deviation of the upper mediastinum and left apical pulmonary cystic features, suggestive of Congenital Cystic Adenomatoid Malformation (CCAM) of the lung. Subsequently, surgical removal of the left upper lobe was performed and CCAM type II was confirmed by histopathology.

Conclusion Although primary spontaneous pneumothorax in adolescents is the most common subtype, in cases where the course of the disease is not as expected, a secondary cause should be considered.

Even though CCAM is considered a rare disorder, it is the most common congenital pulmonary malformation with an incidence of $1: 8300$ to 35.000 live-births. CCAM occurs mostly sporadically and in about $1 / 3$ of the cases the diagnosis is made after the neonatal period. In the presence of pneumothorax, CCAM type IV should be considered and deserves special attention due to its malignant potential. In symptomatic cases, the therapeutic measures consist in pulmonary resection of the affected areas, which is curative and has few complications.

\section{A CASE OF A 13-YEAR-OLD BOY WITH PARAPNEUMONIC EFFUSION CAUSED BY STREPTOCOCCUS CONSTELLATUS}

Charisti Zampetaki*, Ioanna Farakla, Eufrosini Mpriasouli, Theoni Petropoulou. First Department of Paediatrics, National and Kapodistrean University of Athens, Greece, Aghia Sophia Children's Hospital

\subsection{6/archdischild-2021-europaediatrics.425}

A 13-year-old previously healthy male presented with a twoweek history of intermittent fever and left subcostal pain, which had previously been attributed to viral gastroenteritis. He was tachypnoeic, in need of supplemental oxygen, and on examination there was no air entry on the left side of the chest. Laboratory findings included leucocytosis (WBC 27000/ $\mu \mathrm{l}$ with a left shift) and high levels of inflammatory markers (CRP>300 mg/L).

Chest X-ray revealed complete whiteout of the left lung and subsequent ultrasound showed a large loculated pleural effusion. CT chest confirmed the findings and showed the extent of the infection and effusion. The patient was taken to theatre where a chest drain was inserted, automatically draining approximately $500 \mathrm{ml}$ of pus-like fluid, which was sent for cell count, microscopy, and cultures.

The patient was started on broad-spectrum intravenous antibiotics and received intrapleural fibrinolysis treatment with alteplase. His PCR and antibodies for SARS-CoV2 were negative. The pleural fluid analysis confirmed an exudate, and molecular methods as well as pleural fluid cultures indicated Streptococcus constellatus as a cause for his pneumonia and subsequent complicated parapneumonic effusion. Sputum cultures and blood cultures were negative.

Despite optimal antibiotic treatment with cefotaxime and teicoplanin based on sensitivities, there was little clinical and radiological improvement over the next few days, therefore the decision was made for the patient to return to theatre for decortication of the left lung and thoracostomy.

Following this steady progress was made and the patient was able to mobilize and benefit from physiotherapy to help expand the left lung.

Inflammatory markers returned to normal, and imaging showed marked improvement. The patient completed a total of 3 weeks of intravenous antibiotics. The chest drain was removed on day 18 of his hospital stay after having drained a total of over $1500 \mathrm{ml}$ of fluid.

Prior to discharge, as the streptococcus species isolated was not considered typical of the patient's age group and course of illness further tests were sent. Streptococcus constellatus is a group $\mathrm{F}$ beta streptococcus species, known to cause high 